\title{
Various Wolbachia genotypes differently influence host Drosophila dopamine metabolism and survival under heat stress conditions
}

Nataly E. Gruntenko ${ }^{1 *}$, Yury Yu. llinsky ${ }^{1,2,3}$, Natalya V. Adonyeva', Elena V. Burdina ${ }^{1}$, Roman A. Bykov', Petr N. Menshanov ${ }^{1,2,4}$ and Inga Yu. Rauschenbach ${ }^{1}$

From Belyaev Conference

Novosibirsk, Russia. 07-10 August 2017

\begin{abstract}
Background: One of the most widespread prokaryotic symbionts of invertebrates is the intracellular bacteria of Wolbachia genus which can be found in about $50 \%$ of insect species. Wolbachia causes both parasitic and mutualistic effects on its host that include manipulating the host reproductive systems in order to increase their transmission through the female germline, and increasing the host fitness. One of the mechanisms, promoting adaptation in biological organisms, is a non-specific neuroendocrine stress reaction. In insects, this reaction includes catecholamines, dopamine, serotonin and octopamine, which act as neurotransmitters, neuromodulators and neurohormones. The level of dopamine metabolism correlates with heat stress resistance in Drosophila adults.
\end{abstract}

Results: To examine Wolbachia effect on Drosophila survival under heat stress and dopamine metabolism we used five strains carrying the nuclear background of interbred Bi90 strain and cytoplasmic backgrounds with different genotype variants of Wolbachia (produced by 20 backcrosses of Bi90 males with appropriate source of Wolbachia). Non-infected Bi90 strain (treated with tetracycline for 3 generations) was used as a control group. We demonstrated that two of five investigated Wolbachia variants promote changes in Drosophila heat stress resistance and activity of enzymes that produce and degrade dopamine, alkaline phosphatase and dopamine-dependent arylalkylamine N-acetyltransferase. What is especially interesting, wMelCS genotype of Wolbachia increases stress resistance and the intensity of dopamine metabolism, whereas wMelPop strain decreases them. wMel, wMel2 and wMel4 genotypes of Wolbachia do not show any effect on the survival under heat stress or dopamine metabolism. L-DOPA treatment, known to increase the dopamine content in Drosophila, levels the difference in survival under heat stress between all studied groups.

Conclusions: The genotype of symbiont determines the effect that the symbiont has on the stress resistance of the host insect.

Keywords: Drosophila melanogaster, Wolbachia, Dopamine metabolism, Heat stress, Stress resistance

\footnotetext{
* Correspondence: nataly@bionet.nsc.ru

'The Institute of Cytology and Genetics of SB RAS, Novosibirsk 630090, Russia

Full list of author information is available at the end of the article
} 


\section{Background}

The phenomenon of symbiosis can hardly be overestimated: apparently, there is no multicellular organism in nature without symbiotic relations. The crucial issues of symbiosis are the identification of evolutionary stages of establishing interactions between partners and the revealing of molecular mechanisms of these interactions at the cellular and gene level. One of the most widespread prokaryotic symbionts of invertebrates is the intracellular $\alpha$-proteobacteria Wolbachia pipientis that infects no less than a $40 \%$ of terrestrial arthropods [1]. Wolbachia is maternally transmitted and is able to manipulate host sex determination or reproductive systems in order to help Wolbachia spread in host populations [2, 3]. On the other hand, Wolbachia infection could be beneficial to its host [4-8]. Differences in the phenotypical manifestations of the infection can be due to singularities of the host organism physiology, including processes of the endocrine regulation of growth, development and fitness. Indeed, there is a lot of research that connects biochemical changes in hosts and phenomenon of host resistance to viral infections with Wolbachia symbiont [6, 9-12]. However, molecular mechanisms underlying the basis of Wolbachia-host interactions as well as physiological mechanisms by which Wolbachia promotes adaptation of the host organism remain largely unknown. There are several model organisms to study these issues on, and Drosophila melanogaster is the most studied one.

Field populations of $D$. melanogaster are ubiquitously infected with Wolbachia in the frequency range of 30$60 \%$ [13-19]. Wolbachia symbionts of D. melanogaster have monophyletic origin with divergence time $\sim 8$ Kya [20]. Several lineages/genotypes/strains of Wolbachia were identified via different approaches. According to the phylogeny reconstruction of full genome sequences, the symbiont diversity in $D$. melanogaster includes the IVI and VIII clades [20, 21]. In terms of polymorphism of certain genome markers the six genotypes were revealed, i.e. $w$ Mel, wMel2, wMel3, wMel4, wMelCS, wMelCS2 $[18,22]$. Regarding the Wolbachia effect or its source (fly stock), several strains were investigated but two of them (wMel and wMelPop) are the most significant, especially in our discourse. Thus, wMel strain is regarded as a common monophyletic group that covers all of the diversity of bacteria isolated from $D$. melanogaster; whereas wMelPop is a certain pathogenic variant of wMel strain that causes early death of flies [23]. These classifications are consistent as follows: Wolbachia diversity can be reduced to unique, monophelytic wMel strain [24-26], which can be divided into several genotypes, among which wMelPop strain is just a variant of wMelCS genotype. The $w \mathrm{Mel}$ genotype (not to be mixed with wMel strain) includes I-V and VIII clades and is the most widespread $[18,20,27]$. The wMel2 genotype belongs to VIII clade, wMel4 - to III clade, wMelCS and wMelCS2 belong to VI clade [18, 20, 21, 27].

One of the physiological mechanisms that promote adaptation and could be potentially influenced by Wolbachia is a non-specific neuroendocrine stress reaction. In insects, it includes several components, such as juvenile hormone, ecdysone, insulin and biogenic amines, in particular - dopamine (DA) [28-30]. DA plays three different roles in Drosophila: a neurotransmitter passing the nerve impulse through the synaptic cleft; a neuromodulator affecting the neighbouring neurons and modifying neurotransmitter action; and a neurohormone that is transported by the haemolymph and acts remotely [31]. Under the stress the DA level in Drosophila rises quickly and steeply, impacting survival [32-34]. The activity of alkaline phosphatase (ALP), an enzyme regulating the pool of DA precursor tyrosine, is shown to decrease under stress following the rise of the DA level that down-regulates it $[35,36]$. As to the basal DA level under normal conditions, it is determined, at least particularly, via DA-dependent arylalkylamine Nacetyltransferase (DAT) activity [37, 38]. Here we study an effect of several Wolbachia genotypes on Drosophila heat stress resistance and the DA metabolism in order to evaluate the role of Wolbachia diversity in the symbiont influence on the host adaptability.

\section{Methods}

\section{Drosophila melanogaster strains and rearing}

To examine Wolbachia effect on physiological and biochemical traits of $D$. melanogaster, the nuclear background of Bi90 isofemale strain and different cytoplasmic backgrounds were used. Bi90 strain was established from wild-caught female of "Bishkek 2004" population and interbred for more than 300 generations, thereby it could be considered a nearly isogenic line. This strain was earlier characterized by Wolbachia infection and mtDNA [18, 39, 40]. One pair of flies from Bi90 strain was isolated to get Bi90 branch, which was treated with tetracycline for 3 generations to make Wolbachia-free $\mathrm{Bi}^{\mathrm{T}}{ }^{\mathrm{T}}$ strain $[6,27]$. $\mathrm{Bi} 90^{\mathrm{T}}$ strain was used in making conplastic strains and as a control in experiments.

Five D. melanogaster strains with different Wolbachia infections were used in the study: Bi90 strain, that harboured wMel [18], and four conplastic strains which had been produced by 20 backcrosses of $\mathrm{Bi}^{\mathrm{T}} \mathrm{T}^{\mathrm{T}}$ males with appropriate source of Wolbachia. Wolbachia donor strains were also characterized for infection (wMelCS, wMel2, wMel4 and wMelPop) and mtDNA [18, 22, 41] (Table 1). Two independent runs were performed to make each conplastic strain, and finally two strains of 'certain Wolbachia'-cytoplasmic/Bi90 nuclear background were created. 
Table 1 Sources of 'certain Wolbachia' infections used in the study

\begin{tabular}{|c|c|c|c|}
\hline $\begin{array}{l}\text { Drosophila } \\
\text { strain }\end{array}$ & $\begin{array}{l}\text { Wolbachia } \\
\text { infection }\end{array}$ & Donor of cytoplasm & Origin of donor strain \\
\hline $\mathrm{Bi}^{\circ} 0^{\top}$ & non-infected & $\begin{array}{l}\text { Bi90, tetracycline } \\
\text { treated for } 3 \\
\text { generations }\end{array}$ & Kyrgystan, 2004 \\
\hline Bi90 Mel & wMel & Bi90 & Kyrgystan, 2004 \\
\hline Bi90 ${ }^{\mathrm{Mel} 2}$ & wMel2 & 42 & $\begin{array}{l}\text { Yuzhno-Sakhalinsk, } \\
2015\end{array}$ \\
\hline $\mathrm{Bi} 90^{\mathrm{Mel} 4}$ & wMel4 & w304 & $\begin{array}{l}\text { Sinai Peninsula, } \\
\text { Egypt, } 2010\end{array}$ \\
\hline $\mathrm{Bi} 90^{\mathrm{CS}}$ & wMelCS & w153 & Uzbekistan, 1989 \\
\hline $\mathrm{Bi} 90^{\mathrm{Pop}}$ & wMelPop & $\begin{array}{l}\text { Iso wmelPop } \\
\text { (high copy) }\end{array}$ & $\begin{array}{l}\text { courtesy of Luis } \\
\text { Teixeira (Instituto } \\
\text { Gulbenkian de } \\
\text { Ciência, Oeiras, } \\
\text { Portugal) }\end{array}$ \\
\hline
\end{tabular}

All strains were kept at $25^{\circ} \mathrm{C}, 12: 12 \mathrm{~h}$ photoperiod, in a standard Drosophila medium (agar-agar, $7 \mathrm{~g} \mathrm{~L}^{-1}$; corn grits, $50 \mathrm{~g} \mathrm{~L}^{-1}$; dry yeast, $18 \mathrm{~g} \mathrm{~L}^{-1}$; sugar, $40 \mathrm{~g} \mathrm{~L}^{-1}$ ). Flies hatched within $3-4 \mathrm{~h}$ were pooled for experiments.

\section{Viability analysis}

Viability analysis under heat stress was designed as follows: 1 day before the experiment, females were separated from males, and 5 flies were placed in a vial (25-48 vials in each group under study). Before and after the experiments, the flies were kept at $25^{\circ} \mathrm{C}$. To determine the viability under heat stress, the vials with flies were transferred from $25^{\circ}$ to $38{ }^{\circ} \mathrm{C}$ for $4 \mathrm{~h}$, and then were returned to $25^{\circ} \mathrm{C}$. $24 \mathrm{~h}$ later surviving flies were counted and survival rates were calculated as the percentage of survivors in each vial.

To estimate the effect of L-dihydroxyphenylalanine (LDOPA) treatment on the stability against heat stress, five 4-day-old female flies were placed in vials (17-42 vials in each group under study) in which the bottom and $1 \mathrm{~cm}$ of the wall were covered with filter paper soaked with $0.5 \mathrm{~mL}$ of the nutrition medium. The medium contained $5 \%$ sucrose, $2 \%$ yeast and 1\% L-DOPA (Sigma-Aldrich, USA). After $48 \mathrm{~h}$, the vials were transferred from $25^{\circ} \mathrm{C}$ to $38{ }^{\circ} \mathrm{C}$ for $2 \mathrm{~h} 45 \mathrm{~min}$, and then returned to $25^{\circ} \mathrm{C}$. Survivors were counted in $24 \mathrm{~h}$.

\section{Enzyme activity assays}

To perform ALP and DAT activity measurements a spectrophotometric method was used. To measure ALP activity, flies (10-50 in each group under study) were homogenised on ice in $0.1 \mathrm{M}$ Tris-phosphate buffer (Sigma-Aldrich, USA), pH 8.6 (1 fly in $20 \mu \mathrm{l}$ ) and centrifuged for $5 \mathrm{~min}$ at $13,030 \mathrm{~g}$. Enzyme activity in the supernatant was determined using $\alpha$-naphthylphosphate as substrate. After centrifugation, the supernatant was transferred to Eppendorf microtube $(1.5 \mathrm{ml}$, Axygen Inc., USA) to which $1 \mathrm{ml}$ of reaction mixture $(100 \mathrm{ml}$ 0.1 M Tris-phosphate buffer, $\mathrm{pH} 8.6,100 \mathrm{mg} \alpha-$ naphthylphosphate, $100 \mathrm{mg}$ fast blue RR salt (Chemapol, Czech Republic), $230 \mu \mathrm{l}$ 10\% MnCl, $230 \mu \mathrm{l} 10 \% \mathrm{MgCl}$, 0.5 g polyvinylpyrrolidone (ICN, Russia), and $2 \mathrm{~g} \mathrm{NaCl}$ ) was added. Incubation was carried out at room temperature in the dark for $25 \mathrm{~min}$, and the reaction was interrupted by the addition of $3 \mathrm{ml}$ of ice-cold distilled water.

To measure DAT activity, flies (10-38 in each group under study) were homogenised on ice in $0.05 \mathrm{M}$ Tris$\mathrm{HCl}$ buffer (Sigma-Aldrich, USA), pH 7.2 (2 flies in $120 \mu \mathrm{l}$ ) and centrifuged $5 \mathrm{~min}$ at 13,030 g. Enzyme activity in the supernatant was determined using DA (SigmaAldrich, Switzerland) as substrate. The components of the reaction mixture were added to a cuvette as follows: $300 \mu \mathrm{l}$ of $0.05 \mathrm{M}$ Tris- $\mathrm{HCl}, \mathrm{pH} 7.2,50 \mu \mathrm{l}$ of acetyl CoA (0.5 mM, Sigma-Aldrich, USA) in $0.05 \mathrm{M}$ Tris- $\mathrm{HCl}$, $\mathrm{pH} 7.2,25 \mu \mathrm{l}$ of $12 \mathrm{mM}$ N-phenylthiourea (Fluka, China) in $0.05 \mathrm{M}$ Tris- $\mathrm{HCl}, \mathrm{pH} 7.2,25 \mu \mathrm{l}$ of $40 \mathrm{mM}$ DA in $0.001 \mathrm{~N} \mathrm{HCl}, 50 \mu \mathrm{l}$ of the supernatant, and $50 \mu \mathrm{l}$ of $2.4 \mathrm{mM} \mathrm{5,5-dithiobis(2-nitrobenzoic} \mathrm{acid)} \mathrm{(Fluka,} \mathrm{USA)}$ in $0.05 \mathrm{M}$ Tris, $\mathrm{pH}$ 7.2. The samples were incubated for $2 \mathrm{~min}$ at room temperature in the dark.

The optical density of the obtained reaction products was measured with a SmartSpec ${ }^{\text {TM }}$ Plus spectrophotometer (Bio-Rad, USA) at $405 \mathrm{~nm}$ (DAT) and $470 \mathrm{~nm}$ (ALP) against the reaction zero point. For ALP activity measurements under heat stress flies were exposed to $38{ }^{\circ} \mathrm{C}$ for $1 \mathrm{~h} 40 \mathrm{~min}$; the optimum exposure time was determined previously [36].

\section{Statistics}

All data are represented as means \pm S.E.M. The falsediscovery rate corrections for multiple comparisons were made when appropriate. The data on ALP activity, DAT activity and fly viability were analyzed by 1 -way ANOVA (Strain - the simple factor) or by 2-way ANOVA (Strain - the 1st simple factor; Heat stress or L-DOPA treatment - the 2nd simple factor). Before performing the ANOVA, a Shapiro-Wilk's W test was used to assess normality of the datasets analyzed. All datasets that failed to meet the assumptions of the ANOVA were transformed prior to analysis. The comparison of the group means was performed with the BenjaminiHochberg stepwise post-hoc test. The results were considered significant at probability level $<0.05$.

\section{Results}

The heat stress impact on viability of $D$. melanogaster infected with different Wolbachia genotypes

The results of an evaluation of the viability after heat stress exposure $\left(4 \mathrm{~h} 38{ }^{\circ} \mathrm{C}\right)$ of 6-day-old Drosophila 
females of wild type strain $\mathrm{Bi}^{\mathrm{T}} \mathrm{T}^{\mathrm{T}}$ (uninfected control) and strains that harboured wMel, wMel2, wMel4, wMelCS and wMelPop Wolbachia variants are presented in Fig. 1. No significant difference was found in the survival rates under heat stress between females with Wolbachia genotypes $w M e l, w M e l 2$ and $w M e l 4$ and uninfected control. On the contrary, the survival of females with wMelPop infection was significantly decreased compared with control females and females with $w M e l, w M e l 2$ and $w$ Mel4 genotypes, whereas females with wMelCS genotype of Wolbachia demonstrated a significant increase of viability under heat stress (Fig. 1; Strain $\left.-\mathrm{F}_{(5211)}=14.05, p \ll 0.00001\right)$.

\section{The effects of various Wolbachia genotypes on $D$.} melanogaster alkaline phosphatase (ALP) activity

ALP converts the inert tyrosine conjugate, tyrosine-Ophosphate, into tyrosine and thus changes in ALP activity usually correlate with changes in the DA level in flies [35, 38]. The ALP activity of 1- and 6-day-old Drosophila females infected with $w M e l, w M e l 2, w M e l 4, w M e l C S$ and wMelPop Wolbachia variants and uninfected $\mathrm{Bi}^{\mathrm{T}} \mathrm{T}^{\mathrm{T}}$ strain were measured under normal and heat stress $(1 \mathrm{~h} 40 \mathrm{~min}$ $38^{\circ} \mathrm{C}$ ) conditions. No statistical significance under normal conditions between the ALP activities of the control uninfected flies and flies with Wolbachia genotypes wMel, $w M e l 2$ and $w M e l 4$ was found (Fig. 2a, b). However, the ALP activities in 1- and 6-day-old flies with wMelPop infection were lower and in 1- and 6-day-old flies with $w M e l C S$ - higher, than in $w M e l, w M e l 2, w M e l 4$ and $\mathrm{Bi} 90^{\mathrm{T}}$ at the same age (for Day 1 - Fig. 2a; Strain $\mathrm{F}_{(5213)}=269.41, \quad p \ll 0.00001 ;$ for Day 6 - Fig. 2b; Strain $\left.-\mathrm{F}_{(5233)}=56.87, p \ll 0.00001\right)$. The significant decrease in ALP activity following heat stress in the females of both ages of every strain under study was demonstrated (for Day $1-$ Fig. 2a; Stress $-F_{(1213)}=$

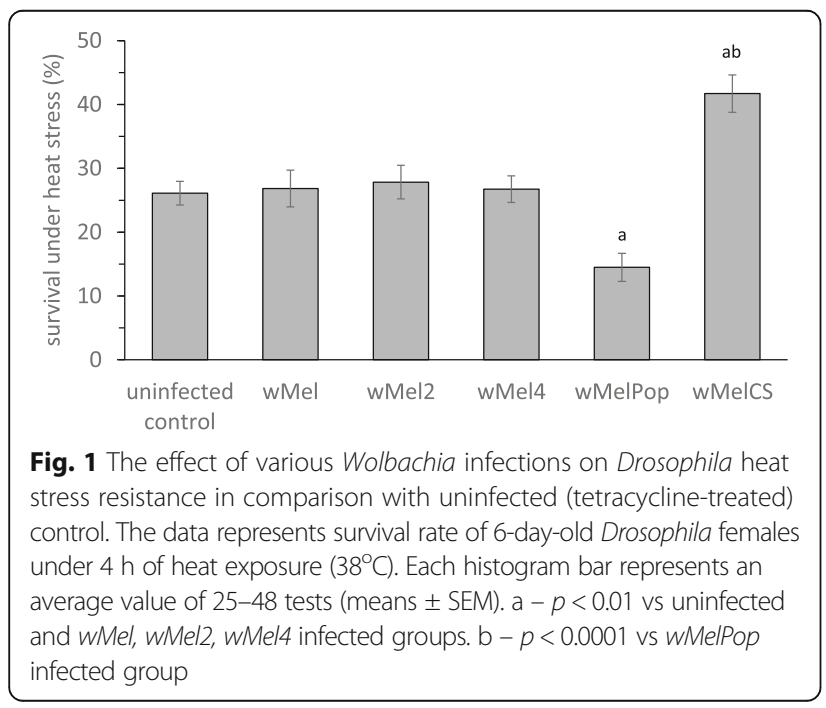

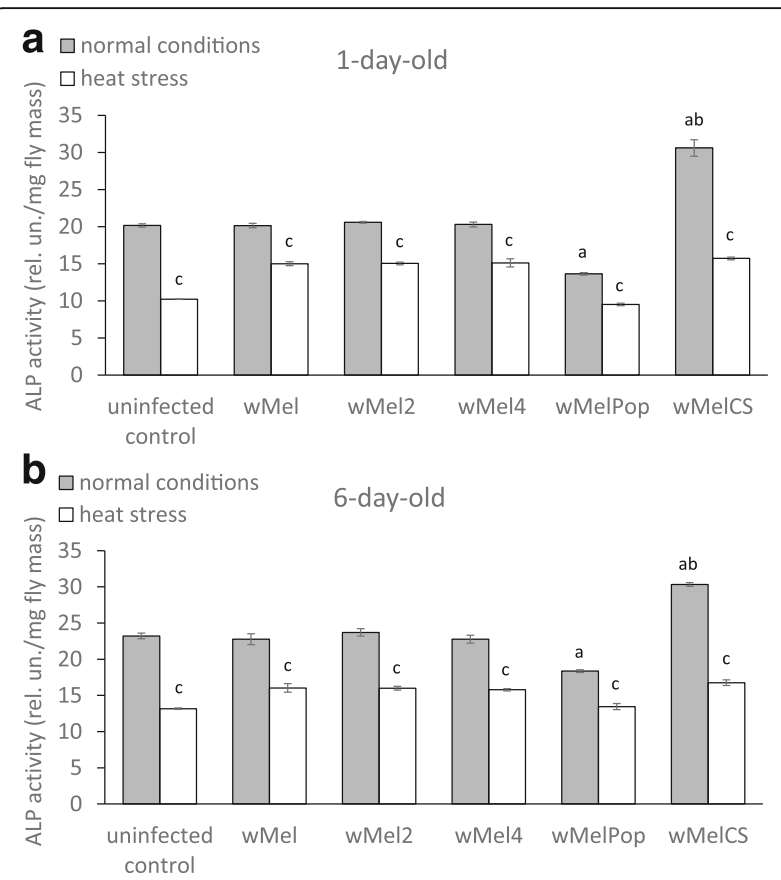

Fig. 2 The effect of various Wolbachia infections on ALP activity in comparison with uninfected (tetracycline-treated) control. a 1-day-old and $\mathbf{b}$ 6-day-old Drosophila females under normal conditions and upon heat stress $\left(38^{\circ} \mathrm{C}\right)$. Each histogram bar represents an average of 10 to 50 measurements (means \pm SEM). $a-p<0.001$ vs uninfected and $w M e l, w M e / 2, w M e / 4$ infected groups that were not stressed. $b-p<0.0001$ vs WMelPop infected non-stressed group. $c-p<0.001$ vs the control non-stressed group of the same strain

$1270.47, \quad p \ll 0.00001 ;$ Strain"Stress $-\mathrm{F}_{(5213)}=74.22$, $p \ll 0.00001$; for Day 6 - Fig. 2b; Stress $-\mathrm{F}_{(1233)}=$ 867.15, $p \ll 0.00001 ; \quad$ Strain*Stress $-\mathrm{F}_{(5233)}=22.22$, $p \ll 0.00001)$.

The effects of different Wolbachia genotypes on $D$. melanogaster dopamine-dependent arylalkylamine $\mathrm{N}$-acetyltransferase (DAT) activity

DAT also takes a part in the regulation of DA content in flies [37], so we measured DAT activities in 1- and 6day-old Drosophila females infected with $w M e l, w M e l 2$, wMel4, wMelCS and wMelPop Wolbachia variants, as well as in the uninfected $\mathrm{Bi}^{\mathrm{T}}{ }^{\mathrm{T}}$ females (Fig. 3). Since DAT does not respond to stress in Drosophila [42], we measured its activity under normal conditions only. The wMelPop infection results in a significant decrease and $w M e l C S$ - in an increase of DAT activity compared with the control in both ages (for Day 1 - Fig. 3a; Strain $\mathrm{F}_{(5147)}=16.06, p \ll 0.00001$; for Day 6 - Fig. 3b; Strain $\left.\mathrm{F}_{(5107)}=16.61, p \ll 0.00001\right)$. Other Wolbachia variants under study do not affect DAT activity in either 1- or 6day-old females. DAT determines, at least particularly, 


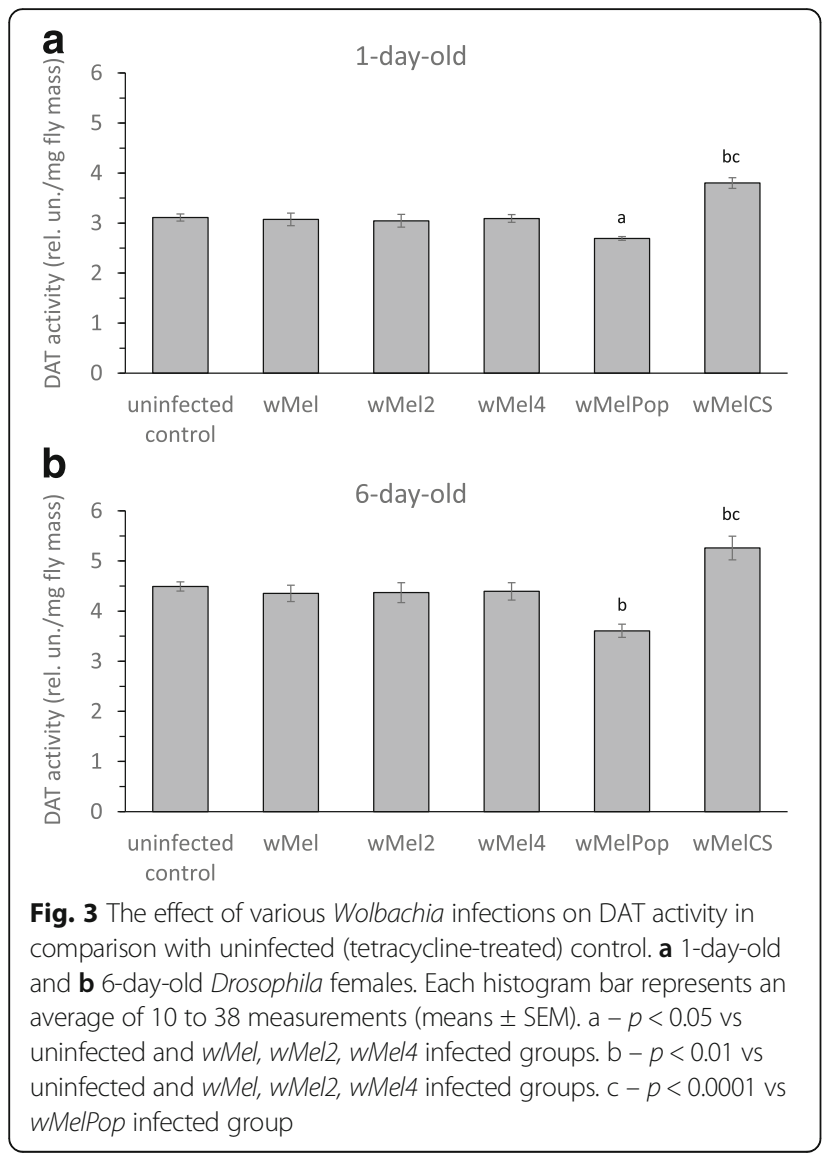

the basal level of DA $[37,38]$, so we have assumed that Wolbachia infection affects it.

The influence of dopamine level on the survival under heat stress of females infected with different Wolbachia variants

To find out whether the changes in the heat stress resistance of Drosophila females infected with wMelPop and $w \mathrm{MelCS}$ have a connection with the altered DA level, we examined their stress resistance following a pharmacological increase of DA content. Feeding with L-DOPA was shown to double the DA level in Drosophila [36], so we fed these females as well as infected with $w \mathrm{Mel}$ and uninfected control with L-DOPA for $48 \mathrm{~h}$ before stress exposure. The rise of the DA level decreases the survival of all four groups under heat stress and eliminates the differences between the survival rate of control and $w M e l$ females and that of females infected with wMelPop and wMelCS (Fig. 4; Strain $-\mathrm{F}_{(3222)}=14.79, p \ll 0.00001$; LDOPA $-\mathrm{F}_{(1222)}=174.18, p \ll 0.00001 ;$ Strain*L-DOPA $\left.\mathrm{F}_{(3222)}=14.86, p \ll 0.00001\right)$. It is worth noting that the survival rates in $\mathrm{Bi}^{\mathrm{T}}{ }^{\mathrm{T}}$, wMel and $w \mathrm{MelCS}$ females after LDOPA feeding do not differ in this parameter from the LDOPA-treated females with wMelPop infection (Fig. 4). The survival rates in $w$ MelPop L-DOPA untreated females

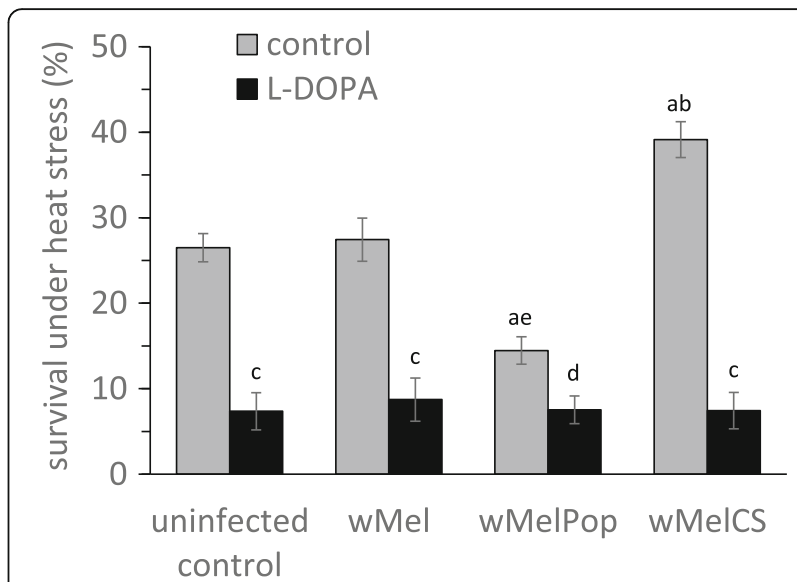

Fig. 4 The effect of L-DOPA on heat stress resistance of Drosophila females with various Wolbachia infections in comparison with uninfected (tetracycline-treated) control. The data represents survival rate of 6-dayold Drosophila females under $4 \mathrm{~h}$ of heat exposure $\left(38^{\circ} \mathrm{C}\right)$ following 2 days of L-DOPA treatment. L-DOPA designates the uninfected flies and flies with various Wolbachia infections that were treated with L-dihydroxyphenylalanine. Each histogram bar represents an average value of 17-42 tests (means \pm SEM). $a-p<0.001$ vs uninfected and wMel infected groups that not received L-DOPA. $b-p<0.0001$ vs WMelPop infected group that not received L-DOPA. $c-p<0.0001$ vs the control group of the same strain that not received L-DOPA. $d$ $p<0.05$ vs the control group of the same strain that not received LDOPA. $e-p<0.05$ vs uninfected and wMel, wMelCS infected groups that received L-DOPA

were low but still higher than in $\mathrm{Bi}^{\mathrm{T}}{ }^{\mathrm{T}}$, wMel and $w \mathrm{MelCS}$ females after L-DOPA feeding (Fig. 4).

\section{Discussion}

Here we try to reveal the influence of the Wolbachia symbiont on heat stress resistance and DA metabolism in D. melanogaster. This investigation was motivated by the reports on Wolbachia effect on insulin signaling $[11,43]$ and data on Wolbachia diversity in D. melanogaster [18, 20-22, 27]. Ikeya et al. [11] had demonstrated the increase of insulin signaling in Wolbachia-infected strains. Insulin signaling pathway interacts with the components of the neuroendocrine stress reaction and the stressresponsive c-Jun-N-terminal kinase (JNK) signaling pathway (which controls of a large number of cellular processes in response a wide range of stressors), and contribute to the fitness and increased stress tolerance $[29,30,44-46]$. The removal of Wolbachia from chico ${ }^{2}$ homozygotes (chico gene codes the Drosophila orthologue of mammalian insulin receptor substrate) resulted in complete lethality [43]. Wolbachia infection was also shown to down-regulate $41 \%$ (11 of 27) of known heat shock proteins in the Drosophila S2 cell line [47].

Using data on D. melanogaster strains with uniform nuclear background but infected with different Wolbachia variants we have shown that Wolbachia genotypes 
wMel, wMel2 and wMel4 (of V, VIII, and III clade, respectively) do not induce alteration in the heat stress resistance and DA metabolism of the host.

However, two Wolbachia isolates under study do cause the changes in survival rate and DA metabolism of $D$. melanogaster host: wMelPop and wMelCS. The wMelPop infection reduces both the survival and activities of ALP and DAT, whereas the $w \mathrm{MelCS}$ infection increases these parameters (see Figs. 2 and 3). Previously, we found the negative correlation of the heat stress resistance with the DA level in Drosophila [48]. The DA level in D. melanogaster is negatively correlated with the level of ALP and DAT activities [36, 38]. Based on this observation, we assumed the DA level to be decreased in the flies with $w M e l C S$ infection (and to be increased in the flies with $w M e l P o p)$. We verified this assumption using the treatment of the flies with the DA precursor, L-DOPA (see Fig. 4). The increase of DA level drastically reduces the survival rates of all studied strains. It is important that increased DA has been revealed to level the viability under heat stress of $w M e l C S$-infected flies with other strains (see Fig. 4).

Low survival under heat stress of the wMelPop-infected flies could be explained by the well-known pathogenicity of this Wolbachia strain [23, 49]. But it is noteworthy that the changes in the DA metabolism are manifested in these flies prior to the mass death of flies (see Figs. 2a and 3a) [23]. The negative effect of wMelPop (in comparison with wMel- and wMelCS-infected and uninfected strains) on the level and the biosynthesis of one more biogenic amine involved in the stress reaction, octopamine, in $D$. melanogaster was shown by Rohrscheib et al. [50]. However, no difference in octopamine biosynthesis pathway was found between flies with wMel and wMelCS Wolbachia genotypes [50]. Perhaps this is due to various roles of DA and octopamine in flies, or with some delicate genetic differences in Wolbachia strains used in our study and in the study of Rohrscheib et al. [50].

We believe that the most interesting result, which we observed here, is the effect of wMelCS Wolbachia on D. melanogaster viability under stress and DA metabolism. Based on the study of Riegler et al. [22] who proposed the hypothesis of global replacement of Wolbachia wMelCS infection by $w \mathrm{Mel}$ in D. melanogaster we expected to find a decreased fitness in wMelCS-infected flies compared with wMel-infected. However, we have found quite the opposite phenomenon.

The design of our study included an attempt to find phylogenetical signal of symbiont effects on the host. Previous works using genome data for both mtDNA and Wolbachia have revealed strict associations of those maternal factors and have distinguished coevolved clades of Wolbachia and mtDNA in D. melanogaster
$[18,20,21,27]$. Here we showed the influence of wMelCS, but not wMel-like, Wolbachia variants on the components of host fitness. The wMelCS-like isolates are related to Wolbachia clade VI that is the most diverged from all other clades, the time divergence of $w M e l$ and $w M e l C S$-like variants is approximately in range 3.2-14 Kya [20]. Thus, we assume a specific influence of Wolbachia clade VI on D. melanogaster that should be verified in the following experiments.

\section{Conclusions}

Here we revealed that the effect of Wolbachia symbiont on the stress resistance and DA metabolism of the host insect depends on the symbiont's genotype variant. We found out that $w \mathrm{MelCS}$ genotype demonstrates a strong positive influence on the D. melanogaster heat stress resistance, while survival rates of the flies with Wolbachia genotypes of $w \mathrm{Mel}$ group do not differ from those of uninfected flies. This result is particularly surprising because genotypes of $w \mathrm{Mel}$ group predominate in the nature populations all over the world and $w M e l C S$ variants are very rare. It is necessary to check whether such a fitness effect is inherent in all wMelCS variants or we are faced with a particular case similar to that of pathogenic wMelPop strain only "with an opposite sign". Besides, we discovered that strong influence of wMelPop strain on D. melanogaster metabolism starts much earlier than mass death of flies.

\section{Abbreviations \\ ALP: Alkaline phosphatase; DA: Dopamine; DAT: DA-dependent arylalkylamine $\mathrm{N}$-acetyltransferase; L-DOPA: L-dihydroxyphenylalanine}

\section{Acknowledgements}

We thank Dr. Luis Teixeira for sharing valuable iso wMelPop strain.

\section{Funding}

The work was supported by the Russian Foundation for Basic Research grant \#16-04-00060 and the State Budgeted Project \# 0324-2016-0002. Publication costs were funded by the Russian Foundation for Basic Research grant \#16-04-00060.

Availability of data and materials

All data analysed during this study are included in this published article.

\begin{abstract}
About this supplement
This article has been published as part of BMC Evolutionary Biology Volume 17 Supplement 2, 2017: Selected articles from Belyaev Conference 2017: evolutionary biology. The full contents of the supplement are available online at https://bmcevolbiol.biomedcentral.com/articles/supplements/ volume-17-supplement-2.
\end{abstract}

\section{Authors' contributions}

IYUR, NEG and YYI conceived and designed the project. YYI and RAB introgressed Wolbachia into the strains. NVA and EVB performed the experiments and collected the data. IYuR and PNM analysed the data. NEG and YYI wrote the paper. All authors gave their approval for this publication.

Ethics approval and consent to participate Not applicable. 


\section{Consent for publication}

Not applicable.

\section{Competing interests}

The authors declare that they have no competing interests.

\section{Publisher's Note}

Springer Nature remains neutral with regard to jurisdictional claims in published maps and institutional affiliations.

\section{Author details}

${ }^{1}$ The Institute of Cytology and Genetics of SB RAS, Novosibirsk 630090, Russia. ${ }^{2}$ Novosibirsk State University, Novosibirsk, Russia. ${ }^{3}$ School of Life Sciences Immanuel Kant Baltic Federal University, Kaliningrad, Russia.

${ }^{4}$ Novosibirsk State Technical University, Novosibirsk, Russia.

Published: 28 December 2017

\section{References}

1. Zug R, Hammerstein P. Still a host of hosts for Wolbachia: analysis of recent data suggests that $40 \%$ of terrestrial arthropod species are infected. PLoS One. 2012;7:e38544.

2. Charlat S, Hurst GD, Merçot H. Evolutionary consequences of Wolbachia infections. Trends Genet. 2003;19(4):217-23.

3. Werren $\mathrm{JH}$, Baldo L, Clark ME. Wolbachia: master manipulators of invertebrate biology. Nat Rev Microbiol. 2008:6:741-51.

4. Fenn K, Blaxter M. Wolbachia genomes: revealing the biology of parasitism and mutualism. Trends Parasitol. 2006;22:60-5.

5. Weeks AR, Turelli M, Harcombe WR, Reynolds KT, Hoffmann AA. From parasite to mutualist: rapid evolution of Wolbachia in natural populations of Drosophila. Keller L, ed. PLoS Biol. 2007:5(5):e114.

6. Teixeira L, Ferreira A, Ashburner M. The bacterial symbiont Wolbachia induces resistance to RNA viral infections in Drosophila melanogaster. PLoS Biol. 2008;6:e2.

7. Osborne SE, Leong YS, O'Neill SL, Johnson KN. Variation in antiviral protection mediated by different Wolbachia strains in Drosophila simulans. Schneider DS, ed. PLoS Pathog. 2009;5(11):e1000656.

8. Versace E, Nolte V, Pandey RV, Tobler R, Schlötterer C. Experimental evolution reveals habitat-specific fitness dynamics among Wolbachia clades in Drosophila melanogaster. Mol Ecol. 2014;23(4):802-14.

9. Hedges LM, Brownlie JC, O'Neill SL, Johnson KN. Wolbachia and virus protection in insects. Science. 2008;322:702.

10. Brownlie JC, Cass BN, Riegler M, Witsenburg JJ, Iturbe-Ormaetxe I, McGraw EA, O'Neill SL. Evidence for metabolic provisioning by a common invertebrate endosymbiont, Wolbachia pipientis, during periods of nutritional stress. PLoS Pathog. 2009;5:e1000368.

11. Ikeya T, Broughton S, Alic N, Grandison R, Partridge L. The endosymbiont Wolbachia increases insulin/IGF-like signalling in Drosophila. Proc R Soc B Biol Sci. 2009;276(1674):3799-807.

12. Faria VG, Martins NE, Magalhães S, Paulo TF, Nolte V, Schlötterer C, Sucena É, Teixeira L. Drosophila adaptation to viral infection through defensive symbiont evolution. Malik HS, ed. PLoS Genet. 2016;12(9):e1006297.

13. Solignac M, Vautrin D, Rousset F. Widespread occurrence of the proteobacteria Wolbachia and partial cytoplasmic incompatibility in Drosophila melanogaster. C R Acad Sci III. 1994;317:461-70.

14. Hoffmann AA, Clancy DJ, Merton E. Cytoplasmic incompatibility in Australian populations of Drosophila melanogaster. Genetics. 1994;136:993-9.

15. Hoffmann AA, Hercus M, Dagher H. Population dynamics of the Wolbachia infection causing cytoplasmic incompatibility in Drosophila melanogaster. Genetics. 1998;148:221-31.

16. Ilinsky Y, Zakharov I. The endosymbiont Wolbachia in Eurasian populations of Drosophila melanogaster. Russ J Genet. 2007;43:748-56.

17. Verspoor RL, Haddrill PR. Genetic diversity, population structure and Wolbachia infection status in a worldwide sample of Drosophila melanogaster and D. simulans populations. PLoS One. 2011;6(10):e26318.

18. Ilinsky YY. Coevolution of Drosophila melanogaster mtDNA and Wolbachia genotypes. PLoS One. 2013;8(1):e54373.

19. Kriesner P, Conner WR, Weeks AR, Turelli M, Hoffmann AA. Persistence of a Wolbachia infection frequency cline in Drosophila melanogaster and the possible role of reproductive dormancy. Evolution. 2016;70:979-97.
20. Richardson MF, Weinert LA, Welch JJ, Linheiro RS, Magwire MM, Jiggins FM, Bergman CM. Population genomics of the Wolbachia Endosymbiont in Drosophila melanogaster. Kopp A, ed. PLoS Genet. 2012;8(12):e1003129.

21. Early AM, Clark AG. Monophyly of Wolbachia pipientis genomes within Drosophila melanogaster: geographic structuring, titre variation and host effects across five populations. Mol Ecol. 2013;22:5765-78.

22. Riegler M, Sidhu M, Miller WJ, O'Neill SL. Evidence for a global Wolbachia replacement in Drosophila melanogaster. Curr Biol. 2005;15:1428-33.

23. Min KT, Benzer S. Wolbachia, normally a symbiont of Drosophila, can be virulent, causing degeneration and early death. Proc Natl Acad Sci U S A. 1997;94:10792-6.

24. Paraskevopoulos C, Bordenstein SR, Wernegreen JJ, Werren JH, Bourtzis K. Toward a Wolbachia multilocus sequence typing system: discrimination of Wolbachia strains present in Drosophila species. Curr Microbiol. 2006;53:388-95.

25. Baldo L, Werren JH. Revisiting Wolbachia supergroup typing based on WSP: spurious lineages and discordance with MLST. Curr Microbiol. 2007:55:81-7.

26. Ros VID, Fleming VM, Feil EJ, Breeuwer JAJ. How diverse is the genus Wolbachia? Multiple-gene sequencing reveals a putatively new Wolbachia supergroup recovered from spider mites (Acari: Tetranychidae). Appl Environ Microbiol. 2009;75:1036-43

27. Chrostek E, Marialva MSP, Esteves SS, Weinert LA, Martinez J, Jiggins FM, Teixeira L. Wolbachia variants induce differential protection to viruses in Drosophila melanogaster: a phenotypic and phylogenomic analysis. Malik HS, ed. PLoS Genet. 2013;9(12):e1003896.

28. Gruntenko NE, Rauschenbach IY. Interplay of JH, 20E and biogenic amines under normal and stress conditions and its effects on reproduction. J Insect Physiol. 2008;54:902-8.

29. Johnson EC, White MP. Stressed-out insects: behavioral modifications and hormonal actions. In: Pfaff DW, Arnold AP, Etgen AM, Fahrbach SE, Rubin RT, editors. Hormones, brain and behavior, vol. 2. 2nd ed. San Diego: Academic Press; p. 1069-96.

30. Gruntenko NE, Rauschenbach IY. The role of insulin signalling in the endocrine stress response in Drosophila melanogaster: a mini-review. Gen Comp Endocrinol. 2017; doi: 10.1016/j.ygcen.2017.05.019.

31. Martin CA, Krantz DE. Drosophila melanogaster as a genetic model system to study neurotransmitter transporters. Neurochem Int. 2014;73:71-88.

32. Gruntenko NE, Chentsova NA, Andreenkova EV, Karpova EK, Glazko GV, Monastirioti M, Rauschenbach IY. The effect of mutations altering biogenic amine metabolism in drosophila on viability and the response to heat stress. Arch Insect Biochem Physiol. 2004;55:55-67.

33. Ueno T, Tomita J, Kume S, Kume K. Dopamine modulates metabolic rate and temperature sensitivity in Drosophila melanogaster. PLoS One. 2012; 7:e31513.

34. Hanna ME, Bednářová A, Rakshit K, Chaudhuri A, O'Donnell JM, Krishnan N. Perturbations in dopamine synthesis lead to discrete physiological effects and impact oxidative stress response in drosophila. J Insect Physiol. 2015;73:11-9.

35. Sukhanova MJ, Grenback LG, Gruntenko NE, Khlebodarova TM, Rauschenbach IY. Alkaline phosphatase in Drosophila under heat stress. J Insect Physiol. 1996:42:161-5.

36. Bogomolova EV, Rauschenbach IY, Adonyeva NV, Alekseev AA, Faddeeva NV, Gruntenko NE. Dopamine down-regulates activity of alkaline phosphatase in Drosophila: the role of D2-like receptors. J Insect Physiol. 2010;56:1155-9.

37. Rauschenbach IY, Adonyeva NV, Alekseev AA, Chentsova NA, Gruntenko NE. Role of arylalkylamine $\mathrm{N}$-acetyltransferase in regulation of biogenic amines levels by gonadotropins in Drosophila. J Comp Physiol B. 2008;178(3):315-20.

38. Rauschenbach IY, Karpova EK, Adonyeva NV, Andreenkova OV, Faddeeva NV, Alekseev AA, Menshanov PN, Gruntenko NE. Disruption of insulin signalling affects the neuroendocrine stress reaction in Drosophila females. JEB. 2014:217:3733-41.

39. Ilinsky YY, Zakharov IK. Cytoplasmic incompatibility in Drosophila melanogaster is caused by different Wolbachia genotypes. Russ J Genet. 2011;1:458.

40. Waisman NY, Golubovsky MD, llinskii YY. Differences in the parameters of longevity and its sex-specificity in human populations and modeling them in Drosophila. Adv Gerontol. 2013;3(4):268-76.

41. Chrostek E, Teixeira L. Mutualism breakdown by amplification of Wolbachia genes. Malik HS, ed. PLoS Biol. 2015;13(2):e1002065.

42. Rauschenbach IY, Sukhanova MZ, Shumnaya LV, Gruntenko NE, Grenback LG, Khlebodarova TM, Chentsova NA. Role of dopa decarboxylase and Nacetyltransferase in regulation of dopamine content in Drosophila virilis under normal and heat stress conditions. Insect Biochem Mol Biol. 1997;27:729-34 
43. Clark ME, Anderson CL, Cande J, Karr TL. Widespread prevalence of wolbachia in laboratory stocks and the implications for drosophila research. Genetics. 2005;170(4):1667-75.

44. Wang MC, Bohmann D, Jasper $H$. JNK signaling confers tolerance to oxidative stress and extends lifespan in Drosophila. Dev Cell. 2003;5:811-6.

45. Karpac J, Jasper H. Insulin and JNK: optimizing metabolic homeostasis and lifespan. Trends Endocrinol Metab. 2009;20:100-6.

46. Gonda RL, Garlena RA, Stronach B. Drosophila heat shock response requires the JNK pathway and phosphorylation of mixed lineage kinase at a conserved serine-proline motif. PLoS One. 2012;7(7):e42369.

47. Xi Z, Gavotte L, Xie Y, Dobson SL. Genome-wide analysis of the interaction between the endosymbiotic bacterium Wolbachia and its Drosophila host. BMC Genomics. 2008;9:1

48. Gruntenko NE, Karpova EK, Alekseev AA, Chentsova NA, Saprykina ZV, Bownes M, Rauschenbach IY. Effects of dopamine on juvenile hormone metabolism and fitness in Drosophila virilis. J Insect Physiol. 2005;51:959-68.

49. Reynolds KT, Thomson $\amalg$, Hoffmann AA. The effects of host age, host nuclear background and temperature on phenotypic effects of the virulent Wolbachia strain popcorn in Drosophila melanogaster. Genetics. 2003;164(3):1027-34.

50. Rohrscheib CE, Bondy E, Josh P, Riegler M, Eyles D, van Swinderen B, Weible MW, Brownlie JC. Wolbachia influences the production of octopamine and affects Drosophila male aggression. Appl Environ Microbiol. 2015;81:4573-80.

\section{Submit your next manuscript to BioMed Central and we will help you at every step:}

- We accept pre-submission inquiries

- Our selector tool helps you to find the most relevant journal

- We provide round the clock customer support

- Convenient online submission

- Thorough peer review

- Inclusion in PubMed and all major indexing services

- Maximum visibility for your research

Submit your manuscript at www.biomedcentral.com/submit 